\title{
Start-Up and Acceleration Profile for Protected Left-Turn Vehicles at Signalized Intersections
}

\author{
Yixin CHEN \\ College of Metropolitan Transportation, Beijing University of Technology, Beijing 100124, China \\ E-mail: chenyixin13@163.com \\ Yulong HE \\ College of Metropolitan Transportation, Beijing University of Technology, Beijing 100124, China \\ E-mail: ylhe@bjut.edu.cn \\ Xiaoduan SUN \\ Civil Engineering Department, University of Louisiana Lafayette, LA 70508, USA \\ E-mail: bjut2003@gmail.com
}

\begin{abstract}
The law of start-up, acceleration profile and leave-out for queuing vehicles at signal intersections is a fundamental question for the queuing theory, traffic simulation and etc in traffic engineering. This paper investigates the vehicular flow at two protected left-turns by video cameras installed at signal intersections, and checks the accuracy of speed processing software. Based on the statistical analysis and the method of curve fitting, the research team establishes the model of start-up and speed-time profile for the queuing vehicles in the protected left-turns at signal intersections. Then the leave-out time of vehicles is calculated based on the established formulas in these models, which is consistent with the observed leave-out time of vehicles by the video. The developed models proved to be accurate. The paper concludes that the queuing vehicles follow by a linear start after the green light, and the speed-time profile indicates an "S" shape from vehicles starting to reach the stable saturation flow. The speed and the queue sequence of first vehicle of saturation flow are different at different intersections, but the law of start-up of vehicles is the same: The same reaction time for the first vehicle and the same linear start-time interval for the adjacent vehicle in the queue. The relationship of leave-out time and the queue sequence of vehicle is nonlinear before the saturation flow. According to the models developed in this paper, the queue sequence of first vehicle of saturation flow and the law of leave-out time of vehicles can be calculated accurately, which is the fundamental for the queuing theory and the traffic simulation.
\end{abstract}

Keywords signal intersection; queuing vehicles; start-up; speed profile model; law of leave-out

\section{Introduction}

Understanding vehicle acceleration and speed variation in a traffic stream is fundamental in traffic flow modeling ${ }^{[1]}$, Vehicle acceleration characteristic provides critical information for the geometric design of intersections, freeway ramps, passing lanes, acceleration lanes, and turning 
bays. Vehicle acceleration models were also used in traffic simulation and fuel consumption models ${ }^{[2-3]}$. However, accurately obtaining vehicle acceleration data at intersections is not an easy task due to the very short acceleration time period for queued vehicles at an intersection. Many data collection techniques and data analysis methodology are not be able to capture the vehiel actual acceleration characteristics in the real world.

Signalized intersections are often the bottleneck of traffic flow. To research for the best congestion control plans through optimizing signal timing design, traffic simulation programs are widely used by transportation system operators and researches alike. The average vehicular delay and the traffic capacity are the main evaluation parameters which needed to be quantified for the optimized strategies made by the traffic simulation ${ }^{[4]}$. The vehicle acceleration characteristics (how they are discharged from the queue)are important input to the models ${ }^{[5]}$. Without the accurate input, no simulation models can produce meaningful results for practical applications.

The researches on the vehicle acceleration profile and discharging characteristics at signal intersections in China are very much limited. In the other countries, researches mostly focus on the acceleration profile models for vehicles. Based on the investigation to the traffic flow by video cameras installed at signal intersections and different video processing, the data of the vehicular start-up time, the speed during the acceleration and the leave-out time for the queuing vehicles are gotten in this paper, then the research team establishes the models of start-up and speed-time profile of queuing vehicles.

According to the actual traffic condition at signalized intersections in China, the flow of protected left-turn vehicles is more stable than that of straight. To exclude the interference of interferential factors, this paper aimed at the protected left-turn lane to develop the models of the start-up and speed profiles for the queuing vehicles.

\section{Literature Review}

When the right-of-way is assigned by traffic signal to vehicles at intersection, the queuing vehicles begin to start one by one. The start-up lost time contains the start-up reaction time of the drivers and the acceleration time of the vehicles. According to the traffic investigation, Zong ${ }^{[6]}$ got the average start-up lost time of the first vehicle is $2.1 \mathrm{~s}$, which is similar to the result of 2.3s in the Fundamentals of Traffic Engineering ${ }^{[7]}$. The software of VISSIM and CORSIM use $2 \mathrm{~s}$ as the start-up lost time ${ }^{[8]}$. In the Highway Capacity Manual ${ }^{[9]}$, the start-up lost time is computed by the sum of the first four vehicles' start-up lost time. Bham and Benekohal ${ }^{[10]}$ got $1.2 \mathrm{~s}$ as the average reaction time in the Ohio Data.

A linear model of the vehicular start time is developed by Zong ${ }^{[6]}$ and Yang ${ }^{[11]}$ : The start time of the vehicle increases linearly with the sequence of the vehicle in the queue. When the vehicles reach the saturate flow, the time of the vehicle to leave the stop line is also linear with the order of the vehicle in the queue ${ }^{[6]}$.

Historical researches used to build the model of speed-time profile based on the accelerationtime profile. This has focused on four types of acceleration characteristic: (a) constant acceleration model (single regime model), (b) dual-regime acceleration model, (c) linear decreasing acceleration model and (d) polynomial acceleration model. 


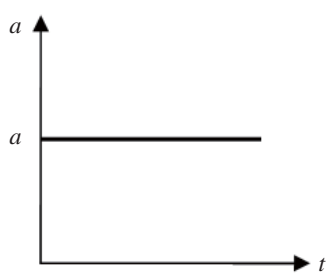

(a)

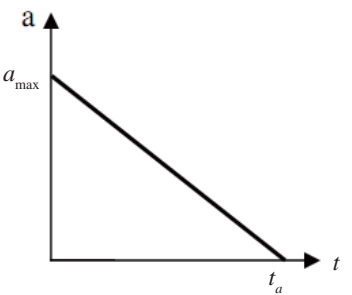

(c)

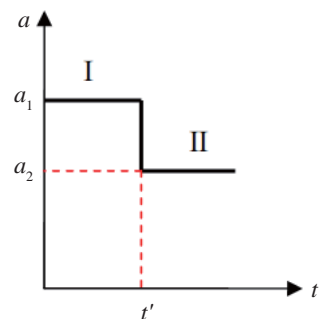

(b)

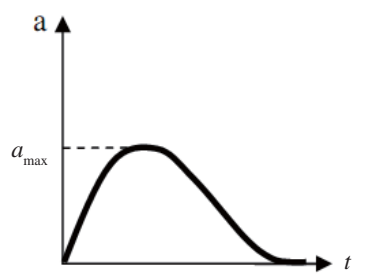

(d)

Figure 1 Acceleration profiles for (a) constant, (b) dual-regime acceleration, (c) linearly decay, and (d) polynomial models

Constant acceleration model assumes a constant rate of acceleration throughout the acceleration maneuver ${ }^{[12]}$. In that model, the speed of vehicles tends to change abruptly. For the vehicles in cellular automata model, they can come to a stop from a maximum speed of $135 \mathrm{~km} / \mathrm{h}$ in one second. NETSIM, FRESIM, and CARSIM used the constant acceleration models also ${ }^{[9]}$.

Several studies demonstrated that the assumption of a constant acceleration rate is unrealistic for through acceleration. It is conventional that the acceleration is greater when speed is low, and smaller when speed is high ${ }^{[13]}$. Bham and Benekohal ${ }^{[10]}$ selected the average speed profile of vehicles from the field data and presented the dual-regime model providing higher acceleration rate at lower speeds and lower acceleration rate at higher speeds (Figure 1(b)). The sets of field data are used in that paper were from the Ohio State University Data (1975) and the FHWA Data ${ }^{[14]}$. Both the data sets provide speed of vehicles at one second interval. For Ohio Data, the speed of the vehicle is estimated to be accurate within $\pm 1.6 \mathrm{~km} / \mathrm{h}$.

The linearly-decreasing acceleration model assumes that the maximum acceleration rate occurs at the beginning of the acceleration process while vehicle speed is zero, and decreases to zero at the end of the acceleration process while vehicle speed reaches free flow speed. Research shows that the linearly-decreasing acceleration model provides a good representation of normal acceleration characteristics except for the unrealistic assumption of high initial acceleration rate at the beginning of the acceleration process ${ }^{[15]}$.

Akcelik and Biggs ${ }^{[1]}$ and Akcelik and Besley ${ }^{[16]}$ proposed a nonlinear polynomial acceleration model to describe the relationship between acceleration rate and time. The researchers collected field data from an instrumented vehicle through urban and non-urban area chase-car studies of 1037 vehicles in Sydney Australia. The results reported that the polynomial model provided good fit to the field data (Figure 1(d)).

Liu et al. ${ }^{[17]}$ and Nie et al. ${ }^{[18]}$ tested the speed profile of the drive shaft of the vehicle by the rotary transducer and obtained the speed after the vehicles start up. The findings showed 
that the acceleration becomes slower after the first fast. Using the second-by-second speed data collected by the Sydney University, Mechanical Engineering Department, by means of an instrumented car in urban, suburban and rural road condition ${ }^{[19]}$, Akcelik ${ }^{[1]}$ selected the acceleration as the variable, and evaluated comparatively three models of acceleration (a twoterm sinusoidal, a three-term sinusoidal, and a polynomial model). The results showed that the polynomial model was the best for predicting acceleration distance. For the speed profile, the authors found that these models yield the S-shaped speed-time trace, but authors didn't present the model of speed profile.

Generally, there are four major variables involved in the acceleration process: speed, time, rate of acceleration, and distance. In this study, speed and time are the original information collected from the field. As a result, these two variables exhibit better accuracy than secondary data, such as the rate of acceleration, which is ultimately calculated using the speed and time. Distance is an accumulative variable, and can therefore result in confounding errors thereby decreasing data accuracy. Thus, the majority of researcher focused on the speed vs. time relationship, which is the speed profile instead of the acceleration profile, to model the acceleration process.

The accuracy of speed-time profile for the vehicles after start-up is largely limited to the tools or the methods that used to survey the vehicular speed. The acceleration process only occurs for short time periods throughout the natural driving process. This makes it difficult to capture natural driving behaviors and to investigate the influence of road physical features on the acceleration process. Historically, researchers performed various vehicle acceleration characteristics studies dating back decades, and developed various types of models to describe the acceleration process. However, the data used in previous studies are usually characterized by limited data collection techniques and methodologies. Database used in this study was usually investigated by in-vehicle Global Positioning System (GPS) technology, and the measurement error of GPS data is about $\pm 5 \mathrm{~m}$, about $5 \mathrm{~km} / \mathrm{h}^{[20]}$. The speed and acceleration data investigated by the instrumented vehicle can be affected by the type of the instrumented vehicle or the personal characteristics of the driver. The laser speed gun was often used to investigate point speed, it is not suitable to get the continuous speed. In this case, the video surveys are used more and more widely because it is labor-saving and accurate, such as the velocities acquired by the aerial photography were determined within $1.6 \mathrm{~km} / \mathrm{h}$. However, video surveys collected may limited by the influence factors of angle of view and the place.

For the acceleration model of the queuing vehicles at signal intersections, the researchers mostly focused on the leading passenger car. E.g., Chowdhury and Rao ${ }^{[21]}$ proposed a twophase linearly-decreasing acceleration model based on field data collected from a signalized intersection for the leading passenger car in a queue. Long ${ }^{[22]}$ made an extensive discussion and study of acceleration characteristics of starting vehicles. He mentioned previous research that was carried out at signalized intersections decades ago using data from spot studies. The researches on the law of start-up, acceleration profile and leave-out for queuing vehicles at signal intersections in China is less.

Based on the investigation of the traffic flow by video cameras installed at signal intersections and using different video processing, the research team checked the accuracy of speed processing 
software and established the models of start-up and speed-time profile for the queuing vehicles. The database was investigated from the passenger cars without the effects of pedestrians in protected left-turn at urban signal intersections.

\section{Data Collection}

In order to ensure the credibility of the study and make compare with different intersections, the imagery data have been collected by the camera placed at corners of two intersections in order to get the accurate bird-view. These typical urban four-approach intersections operate with a four-phase signal plan with a protected left-turn phase for both heavily traveled streets. During the rush hours, majority of left turning vehicles have to wait for more than one signal cycle to pass the intersection. The information for the investigated intersections is shown in Table 1.

Table 1 Information for the investigated intersections

\begin{tabular}{ccc}
\hline Collection site & Intersection A & Intersection B \\
\hline Width of left-turn lane & $3.3 \mathrm{~m}$ & $3.2 \mathrm{~m}$ \\
Length of left-turn lane by video observation & $50 \mathrm{~m}$ & $75 \mathrm{~m}$ \\
Traffic volume of whole intersection (7:00-19:00) & $52,500 \mathrm{pcu}$ & $41,400 \mathrm{pcu}$ \\
Average queue vehicle number & $>8$ & 8 \\
The max queue vehicle number & $>12$ & 14 \\
\hline
\end{tabular}

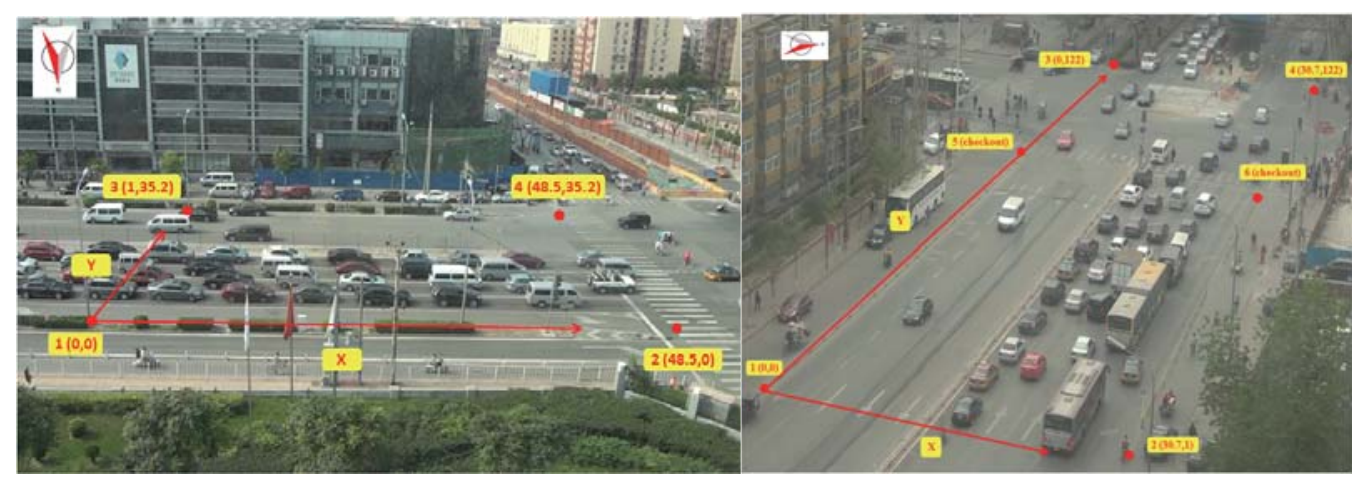

(a)

(b)

Figure 2 Layout of the investigated intersections

The data collection lasted for three consecutive workdays at each of the two investigated signal intersections. With the collected imagery data and the spatial data from processing software, the following variables are obtained: Start-up time of the queuing vehicles after the green light, the speed of the vehicular acceleration process from start-up to leaving the stop line, which will be called the speed-time profile of the vehicle. The start-up time of the vehicles is calculated from the field video which is played one by one frame (the time interval is $0.04 \mathrm{~s}$ ). The speed is obtained by a position software every half second, based on the coordinate system illustrated by a Total Station (Figure 2). The data are collected under good weather with 
only small vehicles (excluding the data from the protected phase when buses, large trucks and U-turn vehicles are present) for protected left-turn, in order to obtain the start-time and speed profile accurately.

According to the conclusions of literature views, the accuracy of speed is largely affected by the tools or the methods used in investigation. The accuracy for the measurement error of speed is tested, which is showed in Figure 3.

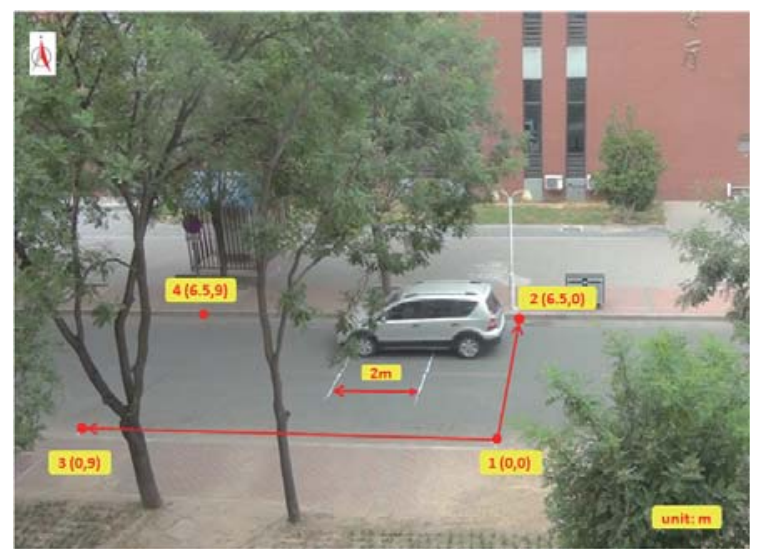

Figure 3 Accuracy test for the measurement error of speed

Two lines are painted on the ground $\left(l_{1}, l_{2}\right)$, and the distance between them is two meters $\left(L_{12}\right)$. The time $\left(T_{12}\right)$ that vehicles pass through $l_{1}$ and $l_{2}$ can be investigated when the field video is played one by one frame (the time interval is $0.04 \mathrm{~s}$ ). Then the actual speed can be calculated by $L_{12}$ and $T_{12}$. The difference between the actual speed $\left(V_{1}\right)$ and the speed $\left(V_{2}\right)$ obtained by the positioning software is the measurement error. The results of accuracy test are showed in Table 2. The average difference between $V_{1}$ and $V_{2}$ is $0.1 \mathrm{~km} / \mathrm{h}$, and the max is $\pm 1.2 \mathrm{~km} / \mathrm{h}$, the relative difference of the speed is within $5 \%$. Thus, the accuracy of the speed investigated by the positioning software is within $\pm 1.2 \mathrm{~km} / \mathrm{h}$ and the relative difference is within $5 \%$.

Table 2 Measurement error of speed

\begin{tabular}{ccccccc}
\hline Speed & Mean & Standard deviation & Min & Max & Median & Sample size \\
\hline$\Delta \mathrm{Vkm} / \mathrm{h}$ & 0.1 & 0.5 & -1.1 & 1.2 & 0.1 & 100 \\
Relative difference & $0.5 \%$ & $2.3 \%$ & $-4.5 \%$ & $4.9 \%$ & $0.5 \%$ & 100 \\
\hline
\end{tabular}

\section{Analysis}

\subsection{Law of Start-Up}

The $5 \%$ censored value method is used in data processing to exclude abnormal data: the start-time of the queuing vehicles is ordered from the small to the large, then the minimum value of $2.5 \%$ and the maximum of $2.5 \%$ is deleted. The sample size for the start-time of the queuing vehicles at intersection $\mathrm{A}$ is 200 , and 150 at intersection $\mathrm{B}$, which are statistic in Table 3 . 
Table 3 Statistic results of start-up time

\begin{tabular}{|c|c|c|c|c|c|c|c|c|}
\hline \multirow{2}{*}{ Queue sequence } & \multicolumn{4}{|c|}{ Intersection A } & \multicolumn{4}{|c|}{ Intersection B } \\
\hline & Mean & Max & Min & Standard deviation & Average & $\operatorname{Max}$ & Min & Standard deviation \\
\hline $1(\mathrm{~s})$ & 1.89 & 2.80 & 0.90 & 0.50 & 1.88 & 2.90 & 0.91 & 0.52 \\
\hline $2(\mathrm{~s})$ & 2.92 & 4.30 & 1.51 & 0.57 & 2.96 & 4.30 & 1.70 & 0.58 \\
\hline $3(\mathrm{~s})$ & 3.94 & 5.31 & 2.70 & 0.61 & 3.98 & 5.60 & 2.67 & 0.60 \\
\hline $4(\mathrm{~s})$ & 4.92 & 6.31 & 3.31 & 0.74 & 5.03 & 6.30 & 3.67 & 0.59 \\
\hline $5(\mathrm{~s})$ & 6.02 & 7.52 & 4.11 & 0.78 & 6.04 & 7.01 & 4.70 & 0.55 \\
\hline $6(\mathrm{~s})$ & 6.91 & 8.51 & 5.40 & 0.80 & 7.10 & 8.01 & 5.81 & 0.54 \\
\hline $7(\mathrm{~s})$ & 8.17 & 9.41 & 6.40 & 0.67 & 8.29 & 9.30 & 7.10 & 0.56 \\
\hline $8(\mathrm{~s})$ & 9.34 & 10.42 & 8.82 & 0.52 & 9.47 & 10.12 & 8.30 & 0.62 \\
\hline Sample size & \multicolumn{4}{|c|}{200} & \multicolumn{4}{|c|}{150} \\
\hline
\end{tabular}

The average start-time interval is stable around a certain value (Table 3), a linear regression model is used to describe the law of start-up time of the queuing vehicles (Figure 4). The fitted formulas are as follows:

At intersection A:

$$
T=1.05 \times k_{a}+0.77
$$

At intersection B:

$$
T=1.07 \times k_{b}+0.76
$$

$T$ : Start-time after the green light(s); $K_{a}$ : Queue sequence of the vehicle at intersection $\mathrm{A} ; K_{b}$ : Queue sequence of the vehicle at intersection B.

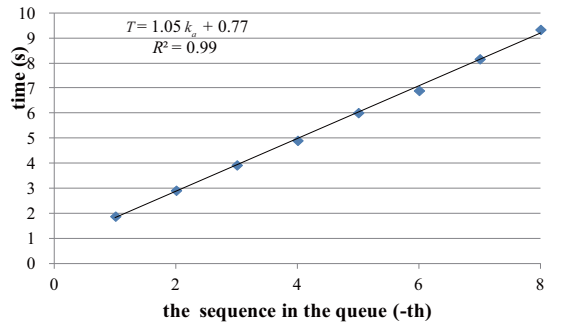

(a)

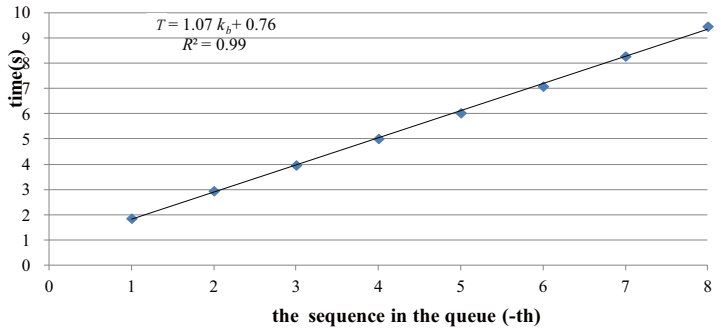

(b)

Figure 4 Regressive analysis between queue sequence and start-up time

The queuing vehicles begin to move one by one after the start-up reaction time, which is showed in Figure 4. The first vehicle's start-up reaction time is the longest, which is $1.82 \mathrm{~s}$ at intersection A: $1.05+0.77$, and it is $1.83 \mathrm{~s}$ at intersection B. There is no difference between them. The mean start-up time between the following vehicles is similar at different intersections, which is $1.05 \mathrm{~s}$ at intersection $\mathrm{A}$ and $1.07 \mathrm{~s}$ at intersection $\mathrm{B}$. 


\subsection{Speed-Time Profile}

The speed of the vehicles after start-up is investigated at the two intersections. Figure 5 shows the scatter diagram of the speed-time profile. The curve is the average speed profile, which is used to establish the speed-time model below.

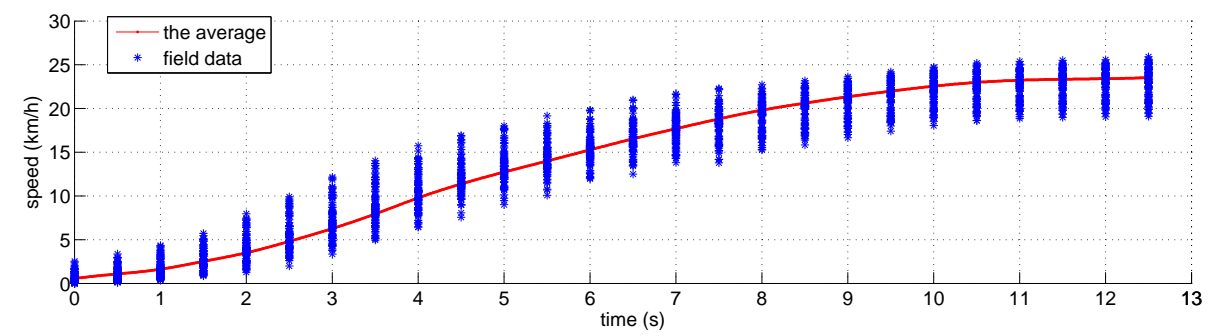

Figure 5 Scatter plot of speed profiles

The following characteristics can be found from Figure 5:

1) Speed profile indicates an "S" shape;

2) Speed is zero at the start and tends to a stable value at the end.

Based on the two characteristics, an improved "S" curve formula $\left(S_{1}+S_{2}\right)$ is used to shape the speed-time profile $(V(t))$. The improved "S" curve formula combines the "S" curve formula which the end is gentle $\left(S_{1}\right)$ and the "S" curve through the origin point $\left(S_{2}\right)$.

Final equation model is as follows:

$$
V(t)=\frac{a_{1}}{1+a_{2} \times \mathrm{e}^{\left(a_{3} \times t\right)}}+\frac{a_{4}}{1+a_{5} \times t^{a_{6}}}
$$

$V(t)$ : Speed of the vehicle after start-up; $t$ : Time; e: Natural logarithms; $a_{1}, a_{2}, a_{3}, a_{4}, a_{5}, a_{6}$ : Constant parameters.

There are two parts in Equation (3). The first part is the formula $S_{1}$ and the second is the formula $S_{2}$. According to the Limit Theorem of Function, the sum of the constant parameters $a_{1}$ and $a_{4}$ is the final stable speed, the saturation speed of the vehicular flow. The speed-time profiles of the queuing vehicles at intersection A and intersection B are modeled by the Fitting Tool Software, which are as follows (Equation (4) is the intersection A, Equation (5) is the intersection B):

$$
\begin{aligned}
& V_{a}(t)=\frac{17.75}{1+17.87 \times \mathrm{e}^{(-0.53 \times t)}}+\frac{6.41}{1+19.08 \times t^{(-2.42)}} \\
& V_{b}(t)=\frac{7.81}{1+62.66 \times \mathrm{e}^{(-0.89 \times t)}}+\frac{12.2}{1+11.33 \times t^{(-2.21)}}
\end{aligned}
$$

The figures of speed profiles and acceleration profiles are shown in the Figure 6 and Figure 7. Both of the fitting correlation coefficients are $0.99\left(R^{2}\right)$. 


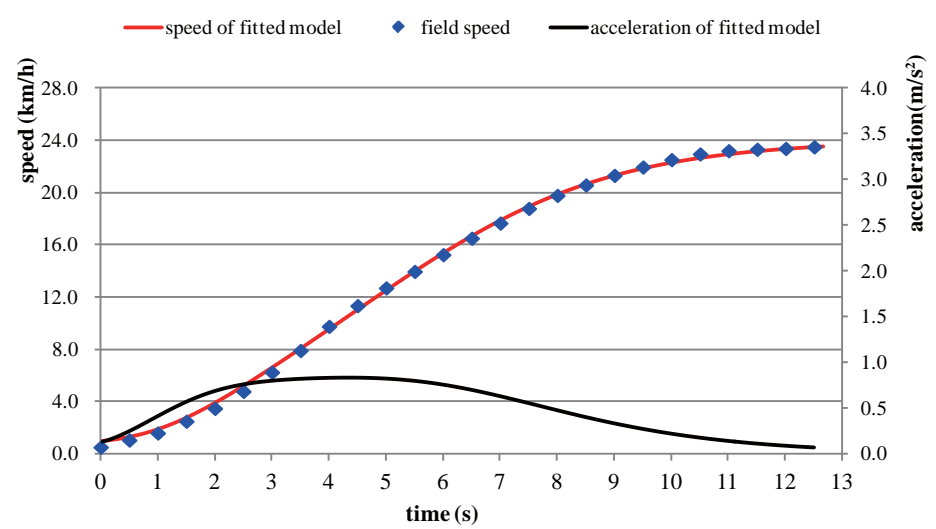

Figure 6 Speed and acceleration profiles (intersection A)

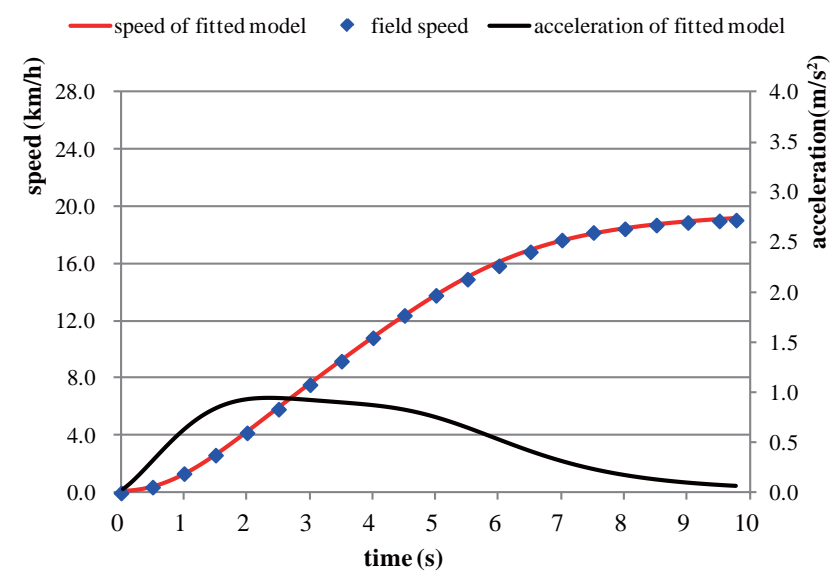

Figure 7 Speed and acceleration profiles (intersection B)

It shows that the S-shaped speed-time model provided good fit to the field data. The speeds increase rapidly at the beginning with a decreasing rate as time increases and end at a relatively stable speed level described as saturation speed. The saturation speed at intersection A is 24 $\mathrm{km} / \mathrm{h}$, and $20 \mathrm{~km} / \mathrm{h}$ at intersection B. The acceleration profile shows that acceleration rate is zero at the start and the end of acceleration run.

\subsection{Law of Leave-Out}

The leave-out time of vehicles is the time when the vehicle passes through the stop line at the intersection. The calculated leave-out time can be solved by the model of start-up and the model of speed profile. By comparing the calculated and the actual leave-out time of the vehicle, the model developed above can be tested, and the model of leave-out time of vehicles can be established accurately also.

Based on the investigated data of the space headway of the still queuing vehicles $\left(h_{d}\right)$ and the statistical analysis, there is no significant difference to $h_{d}$ at different intersections. The $h_{d}$ of both intersection A and B distribute between 5.8 and $7.6 \mathrm{~m}$, and the average of both is $6.6 \mathrm{~m}$ (intersection A is $6.62 \mathrm{~m}$, intersection B is $6.58 \mathrm{~m}$ ). Thus, the time of the vehicles from start-up 
to leaving the intersection can be solved according to the queuing sequence $(K)$ and $h_{d}$, which is denoted as $t_{2}$. The equation used to solve $t_{2}$ is

$$
h_{d} \times k=\int_{0}^{t_{2}} v(t) \mathrm{d} t
$$

The $t_{1}$ is the time of start-up after the green light, which is calculated by Equation (1) or (2) based on the $K$. The sum $t_{1}$ and $t_{2}$ is the leave-out time of vehicles $\left(t_{3}\right): t_{3}=t_{1}+t_{2}$.

With intersection A as an example, Figure 8 is the compare between the actual depart-out time and the calculated by fitted model. The scatters are the actual values and the curve is the calculated.

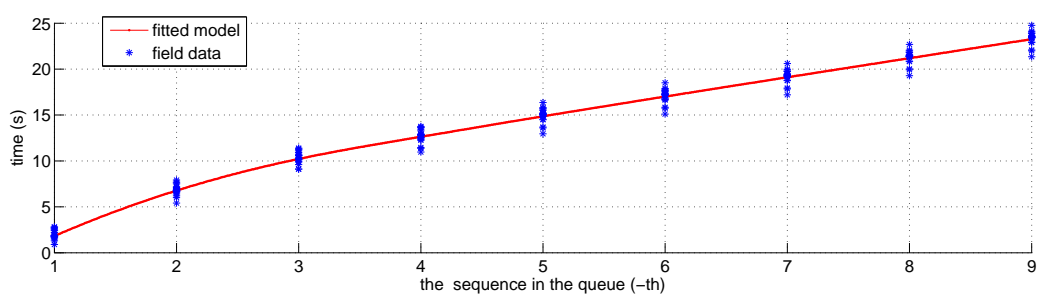

Figure 8 Scatter plot vs. fitted model for the leave time of left-turns

The space headway and the speed of the vehicle increase gradually after start-up. The relationship between leave-time and the queue sequence of vehicles is nonlinear before the saturation flow. Departure rate of the vehicles is linear when the speed of vehicle reaches the saturation speed. It is also shown that the calculated leave-time of vehicles is consistent with the observed actual leave-out time of vehicles. The established models proved to be accurate.

It takes 10.5s when the speed of the vehicle reaches the saturation speed of $24 \mathrm{~km} / \mathrm{h}$ after start-up at intersection A (Figure 6). Let $t_{2}$ equal to $10.5 \mathrm{~s}$, and the distance before saturation flow $(S)$ is

$$
S=\int_{0}^{t_{2}} v(t) \mathrm{d} t
$$

Equation (4) is inserted into Equation (7), and the distance before saturation flow at intersection A $\left(S_{a}\right)$ is obtained: $36.5 \mathrm{~m}$. The queue sequence of first vehicle of saturation flow at intersection A $\left(K_{s-a}\right)$ is $K_{s-a}=S_{a} / h_{d}=5.53$. Thus the sixth vehicle in the queuing is the first to reach the saturation speed. The $K_{S}$ at intersection B can be solved as the same, and the result of $K_{s-b}$ is the fifth vehicle.

\section{Discussion}

The start-up time of the first vehicle is $1.8 \mathrm{~s}$ in this paper, which is similar to the start-up lost time by Zong ${ }^{[6]}$, the Fundamentals of Traffic Engineering and et al. The saturation start-up lost time contains the reaction time and the acceleration time of the vehicles in unsaturation flow, which is $6.6 \mathrm{~s}$ at intersection $\mathrm{A}$ in this paper. It is far greater then $2 \mathrm{~s}$ in VISSIM.

A linear start-up model for the queuing vehicles is developed in this paper (Equations (1) and (2)). However, the constant parameter of reaction time to vehicles is different with other researches. In this paper, the average reaction time to vehicles is $1.06 \mathrm{~s}$, which is close to the $1.2 \mathrm{~s}$ by Bham and Benekohal ${ }^{[10]}$, greater than the result of $0.8 \mathrm{~s}$. The compare between the 
calculated and the actual leave-out time of the vehicle indicates that the average reaction time of $1.06 \mathrm{~s}$ is more realistic.

There are mainly four typical acceleration models for queuing vehicles: Constant, dualregime, linearly decay, and polynomial models. Figure 9 shows the compare between the four typical models. The speed of vehicles tends to change abruptly in constant and two phase models. The highest acceleration is assumed at the beginning of the acceleration process in linearly decay model, which is unrealistic. The polynomial model provides good fit to the field data in the Figure 9. But the speed decreased at the end of the curve, which is also showed in Figure 9. The S-shaped speed-time profile in this paper is realistic relatively. It is similar to the polynomial model of speed profile except for the end part.

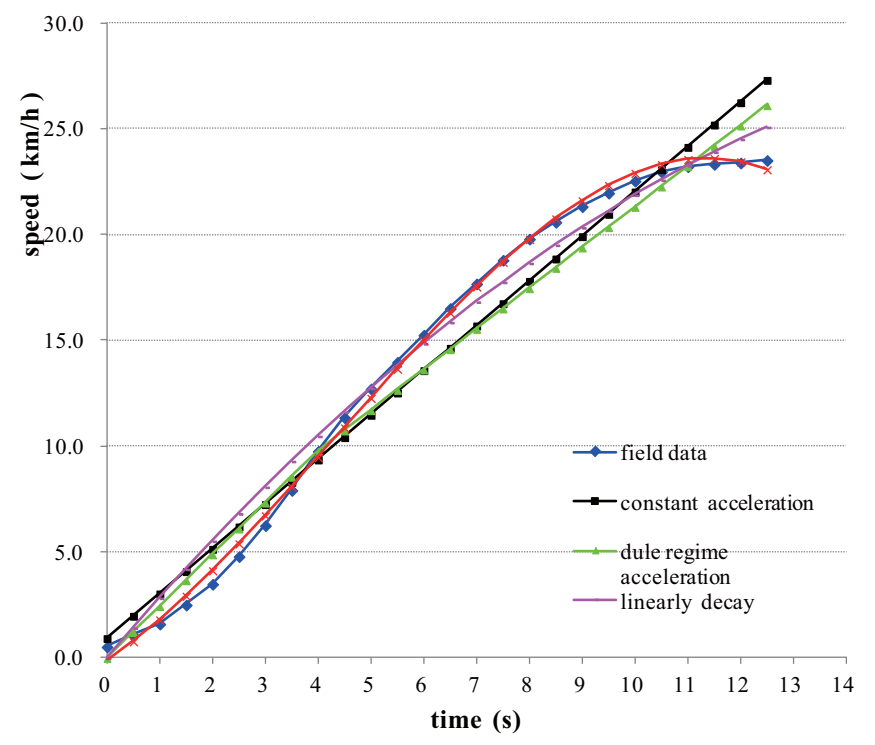

Figure 9 Comparison of speed profiles for different acceleration models

A common problem exists in the four typical acceleration models: They are unable to calculate the speed of saturation low when the parameters are certain in the models. At the end of the curve in the constant, two phase and linearly decay model, the speed is much higher than the actual value, and the speed is smaller than the actual value in the polynomial model (Figure 9). In the S-shaped model of speed-time profile in this paper, the sum of the constant parameters $a_{1}$ and $a_{4}$ is the saturation speed. The saturation speed can be calculated easily by the model itself, and the result tested by the actual field data proved to be accurate.

The speed of saturation flow is different at different intersections, which is mainly affected by the width of the lane and the turning radius. The distance of the vehicle from start-up to reaching the saturation speed is also different. It takes $10.5 \mathrm{~s}$ when the speed of the vehicle reaches the saturation speed of $24 \mathrm{~km} / \mathrm{h}$ after start-up at intersection $\mathrm{A}$, and it takes $9.5 \mathrm{~s}$ when the speed of the vehicle reaches the saturation speed of $20 \mathrm{~km} / \mathrm{h}$ at intersection B. According to the consistent $h_{d}$ of $6.6 \mathrm{~m}$ and the Equation (6), the following results can be gotten. The sixth vehicle in the queuing is the first to reach the saturation speed at intersection $\mathrm{A}$ and the 
fifth at intersection B, which is coincident with the conclusion that the first four vehicles in the queue counter headways longer than the saturation headway in Highway Capacity Manual 2010 edition.

The linear model of leave-out to the queuing vehicles is widely used in queuing theory in traffic engineering, such as the "Traffic Engineering", "HCM2010". The conclusion in this paper is that the leave-time and the queue sequence of vehicles is nonlinear before the saturation flow. When the speed of vehicle reaches the saturation speed, departure rate of the vehicles is linear, which is coincident with the actual (Figure 8).

\section{Conclusion}

By investigating how the queuing vehicles start up and accelerate at two intersections, validation at the microscopic level has been conducted for speed of individual vehicles. The paper concludes that the queuing vehicles follow by a linear start after the green light, and the speed-time profile indicates an "S" shape from vehicles starting to reaching the stable saturation flow. The speed and the queue sequence of first vehicle of saturation flow are different at different intersections, but the law of start-up of vehicles is the same. The relationship of leave-time and the queue sequence of vehicles is nonlinear before the saturation flow. The main methods are statistical analysis and the method of curve fitting.

The accuracy of the software used in this paper for the measurement error of speed is tested, and the actual leave-time of the vehicles from the video is compared with the calculated by the models developed in this paper. There results show close agreement to the field data, which prove the accuracy of the models.

The modeling of the acceleration and speed profiles of vehicles in a traffic stream is a fundamental question in traffic. Vehicular acceleration characteristic provides critical information for the design of intersections and the traffic simulation. At the same time, the law of start-up and leave-out for queue vehicles are the fundamental for the queuing theory in traffic engineering.

The saturation speed is different at different intersection and the acceleration profiles or the speed profile is also different for queuing vehicles. The S-shaped speed profile can be modeled when the saturation speed and the acceleration time are certain, then the trajectory and speed of individual vehicle in microscopic level can be conducted easily. The models developed in this paper will be applied more widely if determining the impact on the saturation speed by the width of the lane and the turning radius, which mainly affected the saturation speed at intersections. It needs more investigated field data to do that work. The imagery data collected by a camera from a bird's-eye-view are limited by the influence factors of angle of view, place and etc. at the intersections. Two feasible intersections are selected to be the database based on a general investigation in this paper.

\section{References}

[1] Akcelik R, Biggs D C. Acceleration profile models for vehicles in road traffic. Transportation Science, 1987, 21(1): $36-54$.

[2] Zhu H. Normal acceleration characteristics of the leading vehicle in aqueue at signalized intersections on arterial streets. The Degree of Master of Science in Civil Engineering, Oregon State University, 2007.

[3] Bokare P S, Maurya A K. Acceleration modeling of vehiclesin developing countries. 2nd International 
Conference on Models and Technologies for Intelligent Transportation Systems, 22-24 June, 2011, Leuven, Belgium, 2011: 1-5.

[4] Qiao J, Xuan H Y, Jiang J H. Micro-simulation analysis for capacity of intersection under different control methods. Journal of Systems Science and Information, 2006, 1: 123-130.

[5] Zhou S Y, Fang J, Yang J. Study on simulation model and control system at multi-phases signalized intersections. Journal of Highway and Transportation Research and Development, 2006, 23(2): 137-140.

[6] Zong E K. Research on capacity of left-turn lanes at signalized intersection. Beijing University of Technology, Beijing, 2012.

[7] Xu J Q, Chen X W. The fundamentals of traffic engineering. The 3rd Edition, China Communication Press, Beijing, 2008, 120-121.

[8] Bloomberg L, Dale J. A comparison of the VISSIM and CORSIM traffic simulation models. The Institute of Transportation Engineers Annual Meeting, 2000.

[9] Transportation Research Board. Highway Capacity Manual. National Research Council, Washington. D. C: National Research Council, 2010.

[10] Bham G H, Benekohal R F. Development, evaluation, and comparison of acceleration models. Transportation Research Board, 81st Annual Conference, Washington, D.C., 2002.

[11] Yang L H, Li L L, Chen H R. Traffic micro-simulation on signalized intersection in urban street. Journal of Harbin Institute of Technology, 2006, 38(8): 1239-1242.

[12] Aycin M F, Benekohal R F. Linear acceleration car-following model development and validation. Transportation Research Record, 1998, 1644(1): 10-19.

[13] Kuang X Y, Xu L H, Wu Y F. Microscopic simulation of signalized intersection based on multi-agent. Computer Engineering and Applications, 2007, 43(19): 217-220.

[14] Smith S A. Freeway data collection for studying vehicle interaction-technical report. Report No. FHWA/RD-85/108, US, Department of Transportation, 1985.

[15] Rakha H, Snare M, Dion F. Vehicle dynamics model for estimating maximum light-duty vehicle acceleration levels. Transportation Research Record, 2004, 1883(1): 40-49.

[16] Akcelik R, Besley M. Micro simulation and analytical methods for modeling urban traffic. Paper presented at the Conference on Advance Modeling Techniques and Quality of Service in Highway Capacity Analysis, Truckee, California, USA, July, 2001.

[17] Liu H O, Chen H Y, Yu C H. Research on technique of measuring the vehicle starting speed. Journal of Transducer Technology, 2001, 20(11): 52-54.

[18] Nie X F, Xi J Q, Chen H Y. The test and research of strategy in the starting for city bus. Auto Mobile Science and Technology, 2005, 3(2): 42-45.

[19] Treiterer J. Investigation of traffic dynamics by aerial photogrammetry techniques. Final Report EES278, Transportation Research Center, Department of Civil Engineering, Ohio State University, 1975.

[20] Ko J, Hunter M, Guensler R. Measuring control delay components using second-by-second GPS speed data. Journal of Transportation Engineering. 2008, 134: 338-346.

[21] Chowdhury M L R, Rao S K. Acceleration characteristics of vehicles at asignalized intersection. Journal of the Institution of Engineers (India), Part C: Civil Engineering Division, 1989, 70(1): 35-37.

[22] Long G. Acceleration characteristics of starting vehicles. Transportation Research Board, 79th Annual Conference, Washington, D.C., 2000. 